\title{
INFLUÊNCIA DO PROCESSO DE MISTURA DE PÓS NA PREPARAÇÃO MAGISTRAL DE CÁPSULAS DE IBUPROFENO
}

\author{
Maiane Rosa*, Fernanda Cramer Flores*, Ruy Carlos Ruver \\ Beck ${ }^{* \star *}$, Andréa Inês Horn Adams ${ }^{\star \star * *}$, Cristiane de Bona da Silva ${ }^{\star \star * *}$ \\ Universidade Federal de Santa Maria
}

\begin{abstract}
RESUMO: O objetivo do presente trabalho foi avaliar a influência do processo de mistura de pós na preparação magistral de cápsulas contendo ibuprofeno. Para a mistura dos pós foram utilizados 0 misturador e triturador automático e mistura manual em saco plástico. Depois de estabelecidos os tempos ótimo de mistura, cápsulas de ibuprofeno foram preparadas ( 3 lotes de 40 cápsulas), nas doses de 100 e $200 \mathrm{mg}$. 0 controle de qualidade das cápsulas foi realizado através da determinação do peso médio, doseamento e uniformidade de doses unitárias. Através dos resultados, diferenças entre os dois processos de mistura foram observadas, especialmente para a mistura de pós contendo ibuprofeno na dose de $100 \mathrm{mg}$. Os lotes obtidos pela mistura manual, neste caso, não cumpriram com as especificações em relação à uniformidade de doses. Para as cápsulas contendo ibuprofeno na dose de $200 \mathrm{mg}$ os resultados foram satisfatórios.
\end{abstract}

Descritores: Cápsulas; Ibuprofeno; Mistura.

\section{INFLUENCE OF THE POWDERS MIXING PROCESS IN THE PREPARATION OF IBUPROFEN COMPOUNDING CAPSULES}

ABSTRACT: The aim of this research was to evaluate de influence of the powder mixture process in the compounding of ibuprofen capsules. The mixing of powder was obtained in an automated mixer or manual (in plastic bags), the last one to simulate the procedures adopted in pharmacy compounding. After determination of the optimum mixing time, ibuprofen capsules were prepared (40 capsules/lot, 3 lots), with 100 or $200 \mathrm{mg}$ of drug. The following tests were done to access the quality of capsules: medium weight, assay and uniformity of dosage units. Differences were observed between the two mixing process, especially for the capsules of lowest dosage $(100 \mathrm{mg})$. In this case, the capsules prepared by manual mixture do not met the requirements of uniformity of dosage units. It was not observed differences between the mixing methods in the case of the capsules of $200 \mathrm{mg}$.

Descriptors: Capsules; Ibuprofen; Mixture

\footnotetext{
*Acadêmica do curso de de Ciências Farmacêuticas da Universidade Federal de Santa Maria, Santa Maria, RS.

**Mestranda do Programa de Ciências Farmacêuticas da Universidade Federal de Santa Maria, Santa Maria, RS.

***Professor Adjunto de Departamento de Produção e controle de medicamentos da Universidade Federal do Rio Grande do Sul, Porto Alegre, RS.

****Professoras Doutoras do Programa de Pós Graduação em Ciências Farmacêuticas, Universidade Federal de Santa Maria, Santa Maria, RS.
} 


\section{Introdução}

Historicamente, a manipulação farmacêutica constitui uma parte integral da prática farmacêutica. ${ }^{1}$ De acordo com a RDC n 67/2007 manipulação é um conjunto de operações farmacotécnicas, com a finalidade de elaborar preparações magistrais e oficinais e fracionar especialidades farmacêuticas para uso humano. ${ }^{2}$

A prescrição magistral tem aumentado rapidamente e representa, atualmente, uma importante área da prática farmacêutica. 0 medicamento manipulado tem se apresentado como uma alternativa farmacêutica, sendo preparadas nas farmácias magistrais as mais diversas formas farmacêuticas. Dentre as formas farmacêuticas sólidas de uso oral destacamse as cápsulas, particularmente as cápsulas duras, que por sua facilidade de formulação e versatilidade, permitem 0 preparo magistral de diferentes formulações em doses individualizadas. $^{3}$

Para o desenvolvimento de uma formulação apropriada é necessário que se leve em consideração as características físicas, químicas e biológicas de todas as substâncias ativas e matérias-primas usadas na fabricação do produto, bem como a anatomia e fisiologia do local de administração e absorção. $O$ fármaco e os excipientes utilizados devem ser compatíveis entre si e com a via de administração desejada. ${ }^{1}$

A maioria dos fármacos administrados na forma de cápsulas requer excipientes para obter homogeneidade no enchimento das mesmas, adequar a velocidade de liberação do fármaco, facilitar a produção, aumentar a estabilidade da formulação, facilitar sua identificação e por razões estéticas. Embora tradicionalmente os excipientes sejam vistos como substâncias inertes, atualmente sabe-se que estes podem interagir com o fármaco, promovendo alterações químicas e físicas, havendo a necessidade de se realizarem estudos de pré-formulação.,34

Existem três fatores importantes a serem considerados na formulação dos pós para enchimento de cápsulas gelatinosas duras. Como o enchimento se dá por gravidade, o fluxo do pó é um aspecto importante, pois quanto mais facilmente o pó escoar, melhor será realizada esta etapa do processo. 0 segundo é a ausência de adesão, ou seja, a mistura não deve aderir ao equipamento para facilitar o seu escoamento. $O$ terceiro é a coesividade que é a capacidade da mistura de formar agregados cilíndricos. ${ }^{5}$

Independentemente da escala de produção, para que se obtenha um produto final cuja dose seja precisa e exata, a avaliação dos componentes da formulação é fundamental. Os princípios ativos e excipientes devem ser totalmente misturados de modo que resulte em uma mistura homogênea. Para que haja uma distribuição adequada do fármaco em toda a mistura dos pós, o ideal é que a densidade e o tamanho das partículas de todos os pós sejam similares. $^{3}$

O ibuprofeno é um anti-inflamatório não esteróide (AINE) derivado do ácido propiônico, sendo empregado, principalmente, como analgésico, anti-inflamatório e antipirético, além de ser uma substância reconhecidamente segura no tratamento pediátrico da febre e da dor. ${ }^{6} \mathrm{O}$ fármaco encontra-se disponível comercialmente no Brasil nas formas farmacêuticas de comprimidos, suspensão oral, creme e gel tópico. Além disso, as farmácias de manipulação

Saúde (Santa Maria), v.36, n.2, p. 07-18, jul./dez. 2010. Influência do processo de mistura de pós na preparação magistral de cápsulas de ibuprofeno

ISSN 2236-5834 preparam cápsulas de ibuprofeno nas doses usualmente prescritas.

Em virtude da necessidade de padronização das etapas que envolvem a preparação magistral de cápsulas, especialmente a etapa de escolha do método de mistura a ser empregado para a adequada homogeneidade dos pós, este trabalho objetivou verificar a influência do processo de mistura de pós na preparação de cápsulas de ibuprofeno, incluindo 
os testes para sua avaliação. As cápsulas, após preparação, foram avaliadas em relação ao peso médio, uniformidade de conteúdo e doseamento do fármaco por volumetria.

\section{Material e métodos}

\section{Materiais}

Para o desenvolvimento das cápsulas empregaram-se as seguintes matérias-primas: ibuprofeno (Delaware, Porto Alegre, Brasil, teor declarado de 99,93\%), celulose microcristalina e lactose (Delaware, Porto Alegre, Brasil); croscarmelose sódica (fabricante FMC Corporation, Philadelphia, USA), dióxido de silício coloidal (fabricante Cabot Corporation, Boston USA) e lauril sulfato de sódio (Vetec, Duque de Caxias, Brasil). Os reagentes e solventes empregados apresentaram grau farmacêutico.

\section{Análise quantitativa e qualitativa da matéria-prima de ibuprofeno}

Os testes realizados compreenderam a avaliação visual, a determinação da solubilidade e da faixa de fusão, bem como o doseamento, descritos na monografia do ibuprofeno. ${ }^{7}$

\section{Preparação das cápsulas}

As cápsulas foram preparadas pelo método de nivelamento, em encapsuladora manual (Tecnofarma, Maringá, PR) após a pesagem e mistura dos constituintes (fármaco e excipientes). Foram preparadas 40 cápsulas de cada lote $(n=3)$, nas doses de 100 e $200 \mathrm{mg}$ de ibuprofeno.

A escolha dos excipientes foi realizada a partir de dados obtidos na literatura, sendo selecionados os mais correntemente utilizados. ${ }^{8}$ Os excipientes empregados compreenderam: croscarmelose sódica $(20 \%$, agente desintegrante), dióxido de silício coloidal $(0,8 \%$, deslizante), lauril sulfato de sódio ( $2 \%$, agente molhante), lactose e celulose microcristalina (diluentes, 75 e 25\%, respectivamente, qsp 100\%). As matérias-primas (fármaco e excipientes) foram tamisadas (tamis 70).

Cápsulas $n^{0} 1$ transparentes (Extracaps $®$, Genix) foram empregadas e as quantidades dos constituintes foram determinadas levando-se em consideração a densidade aparente dos pós, determinada, previamente, para as matérias-primas. 0 peso unitário teórico das cápsulas contendo 100 e $200 \mathrm{mg}$ de ibuprofeno correspondeu a 241 e $262 \mathrm{mg}$, respectivamente.

\section{Mistura dos pós}

Empregaram-se dois métodos para realizar a mistura do fármaco e dos excipientes, determinando-se, a partir das condições experimentais utilizadas, o tempo ótimo de mistura. As quantidades de matérias-primas empregadas foram equivalentes a um lote de 40 cápsulas, nas doses de 100 e $200 \mathrm{mg}$ de ibuprofeno.

\section{Mistura mecânica}

Inicialmente, realizou-se a pesagem individual dos excipientes, os quais foram colocados no misturador e triturador Powdermix (modelo 2004, Santos, SP) e submetidos à homogeneização por 3 e 5 minutos. A velocidade empregada na realização do experimento foi de 44 rotações por minuto (velocidade 3 do equipamento). Após cada intervalo de tempo,

Saúde (Santa Maria), v.36, n.2, p.07-17, jul./dez. 2010

Rosa, M.; Flores, F. C; Beck, R. C. R.; Adams A. I. H.; Silva, C. B. 
alíquotas $(n=8)$ retiradas em diferentes locais do misturador (quantidades equivalentes a 250 $\mathrm{mg}$ de ibuprofeno) foram pesadas e procedeu-se o doseamento do fármaco. As análises foram realizadas em triplicata.

\section{Mistura manual}

Após pesagem, o fármaco e os excipientes foram colocados em saco plástico e a mistura foi efetuada mediante agitação manual, em intervalos de tempo de 3, 5 e 10 minutos para as amostras contendo a dose de $100 \mathrm{mg}$ de ibuprofeno, e 3 e 5 minutos para as amostras contendo a dose de $200 \mathrm{mg}$ de ibuprofeno. Após cada intervalo de tempo, alíquotas $(n=8)$ retiradas em diferentes locais do saco plástico (quantidades equivalentes a $250 \mathrm{mg}$ de ibuprofeno) foram pesadas e procedeu-se o doseamento do fármaco. As análises foram realizadas em triplicata.

\section{Controle de qualidade das cápsulas manipuladas}

\section{Peso médio}

A determinação de peso foi realizada segundo critérios estabelecidos 9 , empregando 20 cápsulas para a determinação. Determinou-se o peso do conteúdo de cada cápsula pela diferença de peso entre a cápsula cheia e a vazia. A partir dos valores obtidos, determinaramse 0 peso médio do conteúdo e os limites de variação superior e inferior permitidos, considerando-se um desvio de $\pm 10 \%$ em relação ao peso médio.

\section{Doseamento}

Para a quantificação do ibuprofeno na mistura dos constituintes e nas cápsulas manipuladas, empregou-se método analítico descrito na monografia farmacopéica para comprimidos, com adaptações. ${ }^{7}$ Sendo assim, o mesmo foi revalidado frente aos parâmetros de especificidade, exatidão e precisão.10 Para a validação, preparou-se um lote de 40 cápsulas de ibuprofeno na dose de $200 \mathrm{mg}$, sendo a mistura dos excipientes e do fármaco realizada com auxílio de gral e pistilo, por diluição geométrica. ${ }^{3,8} 0$ doseamento do fármaco nos diferentes lotes foi realizado após a remoção e mistura do conteúdo de 20 cápsulas. Pesou-se o equivalente a $250 \mathrm{mg}$ de ibuprofeno e transferiu-se para frasco erlenmeyer de 125 $\mathrm{ml}$. Adicionou-se $50 \mathrm{ml}$ de etanol e submeteu-se a agitação mecânica por 10 minutos. Após, adicionaram-se três gotas de solução etanólica de fenolftaleína $1 \%(\mathrm{~m} / \mathrm{v})$ e titulou-se com solução de hidróxido de sódio $0,1 \mathrm{M}$ até viragem para rosa. Foi realizado ensaio em branco para as correções necessárias. Cada $1 \mathrm{ml}$ de hidróxido de sódio 0,1 M equivale a 20,628 mg de ibuprofeno. ${ }^{7}$

\section{Uniformidade de doses unitárias}

A uniformidade de dose para as cápsulas contendo $200 \mathrm{mg}$ foi avaliada através da variação de peso, que correlaciona o peso de 10 cápsulas ao teor do lote, obtido no doseamento. Para as cápsulas contendo $100 \mathrm{mg}$ do fármaco procedeu-se a uniformidade de

Saúde (Santa Maria), v.36, n.2, p. 07-18, jul./dez. 2010. Influência do processo de mistura de pós na preparação magistral de cápsulas de ibuprofeno conteúdo, no qual o teor do fármaco é determinado, individualmente, em 10 cápsulas. ${ }^{9}$ Nesse caso, o conteúdo total de cada unidade foi transferido para um frasco, adicionaram-se $25 \mathrm{ml}$ de etanol e submeteu-se à agitação mecânica por 10 minutos. Após, titulou-se com solução de hidróxido de sódio $0,1 \mathrm{M}$, utilizando solução etanólica de fenolftaleína $1 \%(\mathrm{~m} / \mathrm{v})$ como indicador. Foi realizado ensaio em branco para as correções necessárias. 


\section{Resultados e Discussões}

\section{Análise qualitativa e quantitativa da matéria-prima de ibuprofeno}

O ibuprofeno apresentou-se como um pó branco cristalino, praticamente insolúvel em água, facilmente solúvel em etanol, acetona, metanol e clorofórmio, e solúvel em solução alcalina. $\mathrm{O}$ ponto de fusão obtido para a amostra foi de $75,3^{\circ} \mathrm{C} \pm 0,1^{\circ} \mathrm{C}$ (valor de referência 75 a $78^{\circ} \mathrm{C} .{ }^{7}$ Os resultados obtidos atenderam as especificações farmacopéicas.

No doseamento do fármaco, o teor médio foi de $99,86 \%$. O resultado obtido encontra-se de acordo com o limite preconizado para o fármaco, o qual deve estar compreendido entre 97,0 e $103,0 \% .^{7}$

\section{Validação do método analítico}

Na monografia do ibuprofeno na forma de comprimidos apresentada pelas Farmacopéias Americana e Britânica, ${ }^{11,12} 0$ doseamento do fármaco é realizado por cromatografia líquida. 0 método proposto para a quantificação do ibuprofeno nas cápsulas foi baseado nas condições experimentais descritas na monografia da Farmacopéia Brasileira IV, ${ }^{7}$ com modificações. 0 método oficial preconiza a utilização de clorofórmio para a extração do fármaco. Uma vez que o ibuprofeno também é solúvel em etanol, optou-se por empregar etanol para extração, em virtude de sua baixa toxicidade, facilidade de manuseio e diminuição de riscos à saúde do manipulador e ao ambiente.

A interferência dos excipientes na determinação quantitativa do ibuprofeno foi verificada titulando-se soluções contendo os excipientes, na mesma proporção empregada no doseamento das cápsulas. $\mathrm{O}$ volume de solução titulante de $\mathrm{NaOH} 0,1 \mathrm{M}$ gasto nas seis determinações correspondeu ao volume gasto com o branco, o que demonstra que o método proposto é específico, uma vez que os excipientes não interferem na quantificação do fármaco. A precisão do método analítico foi demonstrada através da repetibilidade (intra-dia) e da precisão intermediária (inter-dia). 0 teor de ibuprofeno obtido nas amostras analisadas no mesmo dia foi $99,95 \%$, (DPR 0,27\%) e nas análises realizadas no segundo dia obteve-se 100,13\% (DPR 0,33\%). A precisão intermediária foi avaliada através da determinação do DPR entre os dois dias de análise. $O$ teor médio obtido foi $100,02 \%$, com DPR de $0,30 \%$. Os valores de DPR obtidos (inferiores a 2,0\%) demonstram a precisão do método em termos de repetibilidade e precisão intermediária. ${ }^{10} \mathrm{De}$ acordo com a literatura, o teor de ibuprofeno em comprimidos deve estar compreendido entre $90 \%$ e $110 \%$, empregando a volumetria para a sua quantificação. ${ }^{7} \mathrm{~A}$ partir destes valores de referência, os resultados obtidos neste trabalho encontram-se de acordo com os limites estabelecidos para o ibuprofeno em formas farmacêuticas sólidas orais. A exatidão do método analítico por volumetria apresentou percentual médio de recuperação de 101,34\% (DPR $=1,16)$, o que demonstra a exatidão do método proposto.

A volumetria de neutralização, além de não necessitar de aparelhagem onerosa e ser de fácil execução, demonstrou ser um método específico, preciso e exato para determinação de ibuprofeno em cápsulas, de acordo com as matérias-primas empregadas. Apresenta como desvantagem, no entanto, requerer maior quantidade de substância para análise, devido à sua menor sensibilidade em relação aos métodos instrumentais.

O peso médio obtido para o lote de cápsulas empregado na validação do método foi de 259,4 mg (variações máxima e mínima de 285,3 mg e 233,5 mg, respectivamente). Todas as unidades encontravam-se dentro dos limites estabelecidos.

Saúde (Santa Maria), v.36, n.2, p.07-17, jul./dez. 2010

Rosa, M.; Flores, F. C; Beck, R. C. R.; Adams A. I. H.; Silva, C. B.

ISSN 2236-5843 


\section{Mistura dos pós}

Uma das etapas importantes na preparação de cápsulas se refere à homogeneidade de mistura dos componentes presentes na formulação, a fim de garantir a dose adequada por unidade posológica. A mistura dos pós, em pequena escala, pode ser realizada empregandose 0 gral e pistilo, misturadores automáticos de bancada, ou ainda, a mistura em saco plástico, utilizada em alguns locais.

O controle da homogeneidade da mistura pode ser realizado através de uma inspeção visual para misturas de produtos com cores diferentes entre si, ou pela adição de corante à mistura de pós brancos, bem como pela coleta de um número de amostras em diferentes partes da mistura, quantificando a substância ativa. É importante salientar que o controle visual através da homogeneidade da cor é apenas orientativo ${ }^{8}$.

Neste trabalho empregaram-se dois procedimentos para efetuar a mistura dos constituintes utilizados na preparação das cápsulas de ibuprofeno, nas doses de 100 e 200 mg: (1) mistura mecânica, em misturador automático de bancada, e (2) mistura manual, utilizando o saco plástico. Foram preparadas cápsulas contendo 100 e $200 \mathrm{mg}$ de fármaco, a fim de determinar o tempo ótimo de mistura quando se tem presente diferentes proporções de fármaco na forma farmacêutica. Nas doses de 100 e $200 \mathrm{mg}$, o fármaco corresponde a, aproximadamente, $41 \%$ e $76 \%$ da mistura.

Nas Tabelas 1 e 2 estão expressos os resultados relativos à preparação de 40 cápsulas de ibuprofeno 100 e $200 \mathrm{mg}$, respectivamente, quando submetidos à mistura mecânica e manual. Os resultados foram expressos em percentual, relativo à quantidade teórica de 250 $\mathrm{mg}$ de ibuprofeno.

Para estimar o tempo ótimo de mistura determinou-se o desvio padrão relativo adequado, estabelecendo uma variação de teor do fármaco máxima de $5 \%$ e que $95,5 \%$ do conteúdo das amostras encontre-se dentro destes critérios. $O$ desvio padrão adequado (SE) foi calculado da seguinte forma. ${ }^{5}$

$$
\mathrm{SE}=\mathrm{PA} \times \mathrm{V} / 2
$$

Onde:

$\mathrm{PA}=$ proporção do fármaco na formulação

$V=$ variação de conteúdo médio de $\pm 5 \%$

O valor 2 corresponde a \pm 2 DP, onde 95,5\% das amostras estarão dentro deste limite, seguindo uma distribuição normal (amostras aleatórias). 
Tabela 1 - Teores percentuais de ibuprofeno na mistura de pós para cápsulas de $100 \mathrm{mg}$, obtida pelos processos mecânico e manual (média \pm desvio padrão, $n=8$ )

\begin{tabular}{cccc}
\hline Processo de mistura & & Processo de mistura & \\
Mistura mecânica & 3 minutos & 5 minutos & 10 minutos \\
\hline Lote 1 & $100,61 \pm 3,48$ & $103,31 \pm 0,85$ & ND \\
Lote 2 & $102,41 \pm 2,07$ & $104,72 \pm 0,74$ & ND \\
Lote 3 & $102,96 \pm 1,10$ & $104,92 \pm 0,42$ & ND \\
Média & $101,99 \pm 2,48$ & $104,31 \pm 0,99$ & \\
DPR (\%) & 2,48 & 0,94 & \\
Mistura manual & & & $103,01 \pm 2,25$ \\
Lote 1 & $102,56 \pm 5,15$ & $101,41 \pm 3,26$ & $103,16 \pm 1,34$ \\
Lote 2 & $99,10 \pm 6,95$ & $102,41 \pm 3,14$ & $103,61 \pm 1,62$ \\
Lote 3 & $103,26 \pm 1,19$ & $100,31 \pm 3,18$ & $103,29 \pm 1,72$ \\
Média & $101,64 \pm 5,16$ & $101,37 \pm 3,17$ & 1,66 \\
DPR (\%) & 5,08 & 3,13 &
\end{tabular}

ND: não determinado.

Tabela 2 - Teores percentuais de ibuprofeno na mistura de pós para cápsulas de $200 \mathrm{mg}$, obtida pelos processos mecânico e manual (média \pm desvio padrão, $n=8$ )

\begin{tabular}{ccc}
\hline Processo de mistura & \multicolumn{2}{c}{ Tempo de mistura } \\
Mistura mecânica & 3 minutos & 5 minutos \\
\hline Lote 1 & $101,57 \pm 0,42$ & ND \\
Lote 2 & $101,10 \pm 0,59$ & ND \\
Lote 3 & $101,48 \pm 0,61$ & ND \\
Média & $101,38 \pm 0,56$ & \\
DPR (\%) & $\mathbf{0 , 5 5}$ & \\
Mistura manual & & \\
Lote 1 & $95,61 \pm 1,20$ & $99,58 \pm 0,89$ \\
Lote 2 & $99,40 \pm 1,37$ & $100,53 \pm 2,25$ \\
Lote 3 & $98,60 \pm 2,98$ & $99,25 \pm 1,50$ \\
Média & $\mathbf{9 7 , 8 7 \pm 2 , 0 0}$ & $\mathbf{9 9 , 8 7 \pm \mathbf { 1 , 6 7 }}$ \\
DPR (\%) & $\mathbf{2 , 0 4}$ & $\mathbf{1 , 7 0}$
\end{tabular}

Os valores de DPR indicam que para as cápsulas de $100 \mathrm{mg}$, os tempos ótimos de mistura correspondem a 5 minutos (mistura mecânica) e 10 minutos (mistura manual) (Tabela 1). Para a dose de $200 \mathrm{mg}$, o tempo de mistura de 3 minutos em misturador automático foi suficiente para se obter uma mistura homogênea dos pós, e na mistura manual foram necessários 5 minutos (Tabela 2). De acordo com os resultados obtidos, verificou-se que a mistura dos pós realizada no misturador e triturador automático demonstrou maior eficiência no processo de mistura em um menor tempo quando comparado com a agitação manual em saco plástico, empregando-se uma proporção de fármaco de, aproximadamente, $41 \%$ em relação ao total de constituintes na mistura. 0 processo de mistura em saco plástico apresentou desvantagem em relação à perda de material, uma vez que o pó se dissipa quando o saco é aberto e grande quantidade de pó fica aderida à superfície do plástico.

Saúde (Santa Maria), v.36, n.2, p.07-17, jul./dez. 2010

Rosa, M.; Flores, F. C; Beck, R. C. R.; Adams A. I. H.; Silva, C. B.

ISSN 2236-5843 
O tempo de mistura a que são submetidos os pós é de fundamental importância para uma distribuição homogênea da substância ativa. Obter uma mistura bem definida e homogênea de pós é uma etapa crítica na preparação de cápsulas em farmácias magistrais. Uma mistura de pós ineficaz pode causar um aumento na variação da quantidade de substância ativa nas cápsulas, resultando, geralmente, na reprovação do produto acabado devido à baixa

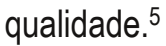

\section{Controle de qualidade das cápsulas manipuladas}

Os resultados obtidos na determinação do peso médio dos lotes preparados para as cápsulas de ibuprofeno nas doses de 100 e 200 mg estão apresentados na Tabela 3.

Tabela 3 - Peso médio dos lotes de cápsulas de ibuprofeno

\begin{tabular}{ccccc}
\hline Processo de mistura & \multicolumn{2}{c}{ Cápsulas $100 \mathrm{mg}$} & \multicolumn{2}{c}{ Cápsulas $200 \mathrm{mg}$} \\
\cline { 2 - 5 } Mistura mecânica ${ }^{1}$ & Peso médio $(\mathrm{mg})$ & DPR $(\%)$ & Peso médio $(\mathrm{mg})$ & DPR $(\%)$ \\
\hline Lote 1 & 237,7 & 3,62 & 251,4 & 4,78 \\
Lote 2 & 242,4 & 2,41 & 251,2 & 2,35 \\
Lote 3 & 237,4 & 1,88 & 254,9 & 1,79 \\
Mistura manual & & & & \\
Lote 1 & 234,3 & 3,97 & 252,8 & 2,58 \\
Lote 2 & 231,4 & 4,86 & 253,8 & 1,73 \\
Lote 3 & 233,5 & 6,58 & 251,7 & 3,44
\end{tabular}

1: 5 minutos para cápsulas de $100 \mathrm{mg}$ e 3 minutos para cápsulas de $200 \mathrm{mg}$.

2: 10 minutos para cápsulas de $100 \mathrm{mg}$ e 5 minutos para cápsulas de $200 \mathrm{mg}$.

O peso médio constitui uma ferramenta essencial para o controle de qualidade de rotina das farmácias magistrais, podendo indicar a ineficiência da técnica de manipulação empregada. A variação individual de peso permitida para cápsulas duras com peso inferior a $300 \mathrm{mg}$ é de $\pm 10 \%$ em relação ao peso médio. 9 Verificou-se que todos os lotes cumpriram as especificações farmacopéicas em relação a este teste. No entanto, observou-se que as cápsulas de ibuprofeno $100 \mathrm{mg}$ referentes aos lotes 2 e 3 (mistura manual) apresentaram maior variação, sendo que uma unidade do lote 2 apresentou peso próximo ao limite superior (peso de 254,1 mg) e duas unidades do lote 3 apresentaram peso abaixo do limite inferior (pesos de 205,2 e 201,2 mg). No entanto, como a Farmacopéia estabelece que podem ser toleradas duas unidades fora dos limites, desde que as mesmas não estejam acima ou abaixo do dobro das percentagens indicadas, todos os lotes foram aprovados neste teste.

Os teores médios percentuais de ibuprofeno obtidos dos 3 lotes de cápsulas na dose de $100 \mathrm{mg}$, preparadas após mistura mecânica e manual, foram 101,90 $\pm 1,27 \%$ e 101,34 \pm $1,94 \%$, respectivamente; para as cápsulas preparadas na dose de $200 \mathrm{mg}$ os valores encontrados compreenderam $98,49 \pm 0,81 \%$ e $97,25 \pm 1,01 \%$, respectivamente. Os resultados obtidos no doseamento das cápsulas de ibuprofeno encontram-se dentro dos valores estabelecidos pela literatura (90 a $110 \%$ ), considerando como referência a monografia do

Saúde (Santa Maria), v.36, n.2, p. 07-18, jul./dez. 2010. Influência do processo de mistura de pós na preparação magistral de cápsulas de ibuprofeno ISSN 2236-5834 ibuprofeno na forma de comprimidos ${ }^{7}$, uma vez que não há metodologia farmacopeica para 0 doseamento de cápsulas de ibuprofeno.

A uniformidade de doses unitárias pode ser determinada por dois métodos: variação de peso e uniformidade de conteúdo. 0 método de variação de peso pode ser aplicado se 0 produto for cápsula mole de conteúdo líquido ou se o produto contiver $50 \mathrm{mg}$ ou mais de um 
componente ativo, compreendendo $50 \%$ ou mais, em peso, da dose unitária da forma farmacêutica. $O$ produto passa o teste se a quantidade de fármaco em 9 das 10 unidades testadas estiver situada entre 85,0 e $115,0 \%$ do valor declarado e nenhuma unidade estiver fora da faixa de 75,0 a $125,0 \%$ do valor declarado, e o DPR obtido deve ser menor ou igual a $6,0 \%$. Em não conformidade com estes critérios, testam-se mais 20 unidades. 0 lote é aprovado se não mais que 3 das 30 unidades testadas estiverem fora da faixa de $85,0 \%$ a $115,0 \%$ do valor declarado e nenhuma unidade fora da faixa de $75,0 \%$ a $125,0 \%$ da quantidade declarada, e o DPR das 30 unidades testadas não for maior que $7,8 \% \cdot{ }^{13}$ Os resultados obtidos nesta avaliação encontram-se representados nas Tabelas 4 e 5.

Tabela 4 - Valores obtidos na determinação da uniformidade de conteúdo (\%) para as cápsulas de ibuprofeno 100 mg, preparadas após mistura dos pós por mistura manual ou mecânica

\begin{tabular}{ccccccc}
\hline Cápsula & \multicolumn{3}{c}{ Mistura mecânica } & \multicolumn{3}{c}{ Mistura manual } \\
& Lote 1 & Lote 2 & Lote 3 & Lote 1 & Lote 2 & Lote 3 \\
\hline 1 & 100,06 & 94,96 & 101,08 & 115,86 & 105,69 & 80,29 \\
2 & 99,04 & 95,98 & 100,06 & 93,50 & 105,69 & 100,61 \\
3 & 98,02 & 97,00 & 98,02 & 110,77 & 108,74 & 95,53 \\
4 & 104,15 & 103,13 & 101,08 & 115,86 & 87,40 & 87,40 \\
5 & 102,11 & 97,00 & 103,13 & 95,53 & 105,69 & 90,45 \\
6 & 101,08 & 100,06 & 101,08 & 92,48 & 88,42 & 85,37 \\
7 & 102,11 & 103,13 & 100,06 & 102,64 & 102,64 & 82,32 \\
8 & 103,13 & 100,06 & 97,00 & 95,53 & 104,68 & 101,63 \\
9 & 101,08 & 95,98 & 101,08 & 104,68 & 103,66 & 92,48 \\
10 & 102,11 & 99,04 & 100,06 & 103,66 & 106,71 & 100,61 \\
Média & 101,29 & 98,63 & 100,27 & 103,05 & 101,93 & 91,67 \\
s & 1,85 & 2,94 & 1,72 & 8,86 & 7,56 & 7,83 \\
DPR (\%) & 1,83 & 2,98 & 1,72 & 8,60 & 7,43 & 8,54 \\
\hline
\end{tabular}

s: desvio padrão.

De acordo com a Tabela 4, as cápsulas de $100 \mathrm{mg}$ de ibuprofeno preparadas pelo processo de mistura mecânica foram aprovados e os valores percentuais de fármaco encontraram-se dentro dos limites especificados. Entretanto, os lotes de cápsulas de $100 \mathrm{mg}$ preparadas pelo processo de mistura manual foram reprovados. Para os lotes 1 e 3 , em duas das 10 unidades testadas, o teor está fora dos limites estabelecidos (85,0 a 115,0\%). Além disso, se verificou grande variação no teor, evidenciado pelos valores de DPR superiores ao permitido $(6,0 \%)^{13}$, nos três lotes. De acordo com relatos de literatura, lotes cujo peso médio apresente DPR superior a 4\% têm grande probabilidade de não serem aprovados no teste de uniformidade de conteúdo. ${ }^{14}$ Sendo assim, considerando a variação desses lotes no peso médio (Tabela 3) e os resultados de uniformidade de conteúdo (Tabela 4), não se realizou reteste dos lotes, o que implicaria em determinar o teor individual em mais 20 unidades. 
Tabela 5 - Valores obtidos na determinação da uniformidade de conteúdo (\%) para as cápsulas de ibuprofeno 200 mg, preparadas após mistura dos pós por mistura manual ou mecânica

\begin{tabular}{ccccccc}
\hline Cápsula & \multicolumn{3}{c}{ Mistura mecânica } & \multicolumn{3}{c}{ Mistura manual } \\
& Lote 1 & Lote 2 & Lote 3 & Lote 1 & Lote 2 & Lote 3 \\
\hline 1 & 96,21 & 100,50 & 97,09 & 99,03 & 99,66 & 95,78 \\
2 & 97,96 & 98,22 & 97,98 & 95,68 & 98,76 & 98,54 \\
3 & 94,19 & 96,76 & 95,81 & 96,90 & 99,54 & 101,15 \\
4 & 100,44 & 97,67 & 99,62 & 100,33 & 97,19 & 100,31 \\
5 & 100,02 & 100,03 & 98,72 & 102,06 & 101,74 & 101,58 \\
6 & 102,15 & 93,89 & 99,62 & 97,77 & 97,78 & 95,94 \\
7 & 100,68 & 100,50 & 100,00 & 101,00 & 101,62 & 92,60 \\
8 & 101,96 & 100,97 & 98,18 & 99,58 & 100,76 & 92,22 \\
9 & 98,31 & 94,80 & 95,81 & 95,68 & 98,40 & 100,54 \\
10 & 95,36 & 101,92 & 98,06 & 97,53 & 96,99 & 92,18 \\
Média & 98,73 & 98,53 & 98,09 & 98,56 & 99,24 & 97,08 \\
s & 2,78 & 2,72 & 1,50 & 2,20 & 1,73 & 3,83 \\
DPR (\%) & 2,82 & 2,77 & 1,53 & 2,23 & 1,74 & 3,95 \\
\hline
\end{tabular}

s: desvio padrão.

Para as cápsulas contendo ibuprofeno na dose de $200 \mathrm{mg}$, todos os lotes avaliados apresentaram resultados satisfatórios no teste de uniformidade de doses realizado pelo método de variação de peso.

Cabe salientar que quando se empregou o misturador e triturador automático, baixos valores de DPR foram obtidos, indicando uma maior eficiência no processo de mistura, resultando em boa homogeneidade entre os teores individuais. De acordo com as condições experimentais empregadas, a mistura manual em saco plástico não é recomendada quando se tem uma proporção de fármaco inferior a $50 \%$ do total de constituintes na formulação. Conforme mencionado anteriormente, este processo de mistura acarretou em perda de material e adesão dos pós ao saco plástico, obtendo-se uma inadequada distribuição dos pós nas cápsulas; entretanto, estes efeitos não comprometeram os lotes preparados na dose de $200 \mathrm{mg}$ de ibuprofeno, em virtude de uma maior quantidade de fármaco em relação à mistura total de pós.

\section{CONCLUSÃO}

A validação do método analítico por volumetria mostrou-se adequado para quantificação de ibuprofeno nas cápsulas manipuladas. A otimização do tempo ótimo de mistura foi de fundamental importância para a preparação de cápsulas magistrais para os métodos selecionados. A utilização do misturador e triturador automático empregado para a mistura dos constituintes da formulação em comparação à mistura manual em saco plástico demonstrou

Saúde (Santa Maria), v.36, n.2, p. 07-18, jul./dez. 2010. Influência do processo de mistura de pós na preparação magistral de cápsulas de maior eficiência em um menor tempo quando se empregou uma proporção de fármaco inferior a $50 \%$ do conteúdo total da mistura. Além disso, se verificou uma maior homogeneidade dos lotes preparados, obtendo-se a aprovação dos mesmos nos testes empregados (peso médio, doseamento e uniformidade de doses) já que nenhuma análise realizada ficou fora das especificações farmacopéicas. Entretanto, a mistura manual dos pós resultou em reprovação 
dos lotes das cápsulas contendo $100 \mathrm{mg}$ de ibuprofeno. Os resultados obtidos neste trabalho evidenciam a importância de padronização dos processos de obtenção de cápsulas magistrais, bem como a necessidade do controle de qualidade de tais preparações.

\section{Referências}

1. Block LH. Medicação tópica. In: Gennaro, AR (ed.) Remington: the science and pratice of pharmacy, (A.R. Gennaro, ed.). Philadelphia: Lippincott Willians \& Wilkins, 2004. P. 862-84.

2. Brasil. Ministério da Saúde. Agência Nacional de Vigilância Sanitária. RDC n 67, de 08 de outubro de 2007. Dispõe sobre boas práticas de manipulação de preparações magistrais e oficinais para uso humano em farmácias. Diário Oficial da União, Brasília, 09 out. 2007. Seção 1.

3. Allen Jr. LV, Popovich NG, Ansel HC. Formas farmacêuticas e sistemas de liberação de fármacos. $5^{\mathrm{a}}$ ed. Porto Alegre: Editora ArtMed, 2007.

4. Jackson K, Young D, Pant S Drug-excipient interaction and their affect on absorption. Research Focus, 2000; 3: $336-45$.

5. Aulton ME. Delineamento de formas farmacêuticas. Porto Alegre. Editora ArtMed, 2005.

6. Drug Evaluation. Ibuprofen. Englewood, Micromedex, Formato eletrônico. (International Healthcare Series for Windows, v. 115); 2003.

7. Farmacopéia Brasileira. São Paulo. Editora Atheneu; 2001.

8. Ferreira AO. Guia prático de farmácia magistral. São Paulo: Editora Pharmabooks, 2008.

9. Farmacopéia Brasileira. São Paulo. Editora Atheneu; 1988.

10. Brasil. Ministério da Saúde. Agência Nacional de Vigilância Sanitária. RDC nº 899, de 29 de maio de 2003. Determina a publicação do "Guia para validação de métodos analíticos e bioanalíticos". Diário Oficial da União, Brasilia, 02 jun. 2003. Seção 1.

11. USP 32. The United States Pharmacopeia. 32 ed. Rockville: United States Pharmacopeial Convention; 2009.

12. BP 2007. British Parmacopoeia. London: The Stationary Office; 2007.

13. Farmacopéia Brasileira. São Paulo. Editora Atheneu; 1996.

14. Pinheiro GM, Benavide VG, Volpato NM, Cabral LM, Santos EP. Indicadores para controle do processo de manipulação de cápsulas em farmácias. Rev. Bras. Farm, 2008; 89(1): 28-31.

Cristiane de Bona da Silva - Av. Roraima, 1000 prédio 26, Centro de

Ciências da Saúde. Telefone: + 5555 3220-8452; Fax: +55 55 3220-8248

E-mail: csbona@smail.ufsm.br

Recebido em 23 de novembro de 2010.

Aprovado em 06 de abril de 2011.

Saúde (Santa Maria), v.36, n.2, p.07-17, 